\title{
Poisson-Lie transformations and generalized supergravity equations
}

\author{
Ladislav Hlavatý ${ }^{1, a}{ }^{\infty}$, Ivo Petr $^{2, b}{ }_{(\mathbb{C}}$ \\ ${ }^{1}$ Faculty of Nuclear Sciences and Physical Engineering, Czech Technical University in Prague, Břehová 7, 115 19 Prague 1, Czech Republic \\ ${ }^{2}$ Faculty of Information Technology, Czech Technical University in Prague, Thákurova 9, 16000 Prague 6, Czech Republic
}

Received: 8 March 2021 / Accepted: 15 May 2021 / Published online: 31 May 2021

(C) The Author(s) 2021

\begin{abstract}
In this paper we investigate Poisson-Lie transformation of dilaton and vector field $\mathcal{J}$ appearing in generalized supergravity equations. While the formulas appearing in literature work well for isometric sigma models, we present examples for which generalized supergravity equations are not preserved. Therefore, we suggest modification of these formulas.
\end{abstract}

\section{Contents}

1 Introduction . . . . . . . . . . . . . 1

2 Basics of Poisson-Lie T-plurality . . . . . . . . 1

3 Poisson-Lie transformations and generalized supergravity equations ................ 3

4 Examples .................... . . 4

4.1 Poisson-Lie plurality on Drinfel'd double (1|5) 5

4.1.1 Identity $(1 \mid 5) \rightarrow(1 \mid 5)$ and full duality $(1 \mid 5) \rightarrow(5 \mid 1) \ldots \ldots \ldots \ldots$

4.1.2 Plurality $(1 \mid 5) \rightarrow\left(6_{-1} \mid i i 5\right) \quad \ldots \ldots .6$

4.2 Poisson-Lie plurality on Drinfel'd double (1|4) 6

4.2.1 Identity $(1 \mid 4) \rightarrow(1 \mid 4) \ldots \ldots . .66$

4.2.2 Full duality $(1 \mid 4) \rightarrow(4 \mid 1) \ldots \ldots .7$

4.2.3 Plurality $(1 \mid 4) \rightarrow\left(6_{-1} \mid i i 4\right) \quad \ldots \ldots .7$

4.2.4 Plurality $(1 \mid 4) \rightarrow\left(i i 4 \mid 6_{-1}\right) \quad \ldots \ldots .7$

5 Conclusions .................. 7

References . . . . . . . . . . . . . . 8

\section{Introduction}

Formula for Poisson-Lie transformation [1] of dilaton field accompanying sigma model background was given long ago in [2]. Its limitations discussed in [3] concern the problem

\footnotetext{
a e-mail: hlavaty@fjfi.cvut.cz

b e-mail: ivo.petr@fit.cvut.cz (corresponding author)
}

of possible appearance of unwanted "dual" coordinates of Drinfel'd double in the transformed dilaton. The problem was partially solved in $[4,5]$ for special cases where transformations of relevant coordinates of Drinfel'd double are linear. The price we had to pay was that in order to keep invariance with respect to Poisson-Lie transformations we had to replace the beta function equations by generalized supergravity equations [6] containing not only dilaton, but also additional vector fields for which correct transformation formulas need to be found. Unfortunately, there are cases for which the transformation of relevant coordinates is not linear or the Poisson-Lie formulas do not provide solutions of generalized supergravity equations.

In the following we have chosen several examples for which the problem of unwanted coordinates in the transformed dilatons does not appear, and still, it turns out that the original formula [5,7-9] for Killing vector field $\mathcal{J}$, which works well for isometric initial sigma models, fails. These are the cases where the initial sigma models are constructed from Manin triple $(\mathfrak{d}, \mathfrak{g}, \tilde{\mathfrak{g}})$ where $\tilde{\mathfrak{g}}$ is neither Abelian nor unimodular. The purpose of this note is to extend the validity of Poisson-Lie formulas to these cases. Beside that for NS-NS generalized supergravity equations it is not necessary to have formulas for both dilaton and Killing vector $\mathcal{J}$ and one only needs formula for Poisson-Lie transformation of one-form $X$ that combines these two. It is given as well.

\section{Basics of Poisson-Lie T-plurality}

Here we shall recapitulate well known basics of Poisson-Lie T-plurality with spectators $[1,2,10]$ to establish notation.

Sigma models in curved background are given by Lagrangian $\left.\mathcal{L}=\partial_{-} \phi^{\mu} \mathcal{F}_{\mu \nu}(\phi) \partial_{+} \phi^{\nu}, \quad \phi^{\mu}=\phi^{\mu}\left(\sigma_{+}, \sigma_{-}\right), \quad \sigma_{ \pm}=\tau \pm ф 1\right)$ where map $\phi$ embeds worldsheet $\Sigma \subset \mathbb{R}^{2}$ into target manifold $\mathscr{M}$ as $\phi: \Sigma \rightarrow \mathscr{M}$. Functions $\phi^{\mu}, \mu=1, \ldots, \operatorname{dim} \mathscr{M}$, 
are compositions of $\phi$ and coordinate map on chart of $\mathscr{M}$. Tensor field $\mathcal{F}=\mathcal{G}+\mathcal{B}$ defines metric and torsion potential (Kalb-Ramond field) of the target manifold $\mathscr{M}$.

Assume that there is $d$-dimensional Lie group $\mathscr{G}$ whose action on $\mathscr{M}$ is smooth, proper and free. The action of $\mathscr{G}$ is transitive on its orbits, hence we may locally consider $\mathscr{M} \approx$ $(\mathscr{M} / \mathscr{G}) \times \mathscr{G}=\mathscr{N} \times \mathscr{G}, \operatorname{dim} \mathscr{M}=\operatorname{dim} \mathscr{N}+\operatorname{dim} \mathscr{G}=n+d$, and introduce adapted coordinates

$$
\begin{aligned}
\left\{x^{\mu}\right\} & =\left\{s_{\alpha}, x^{a}\right\}, \quad \alpha=1, \ldots, n=\operatorname{dim} \mathscr{N}, \\
a & =1, \ldots, d=\operatorname{dim} \mathscr{G}
\end{aligned}
$$

where $x^{a}$ are group coordinates and $s_{\alpha}$ label the orbits of $\mathscr{G}$. Coordinates $s_{\alpha}$ are treated as "spectators" as they do not participate in Poisson-Lie transformations. In this paper we focus on local properties of transformed sigma models and do not impose any conditions following from compactification of $\mathscr{M}$ or $\mathscr{G}$. Discussion of some global issues can be found e.g. in [11] or recent papers [12,13].

Poisson-Lie duality/plurality is based on the possibility to pass between various decompositions of Drinfel'd double $\mathscr{D}$, which is a $2 d$-dimensional Lie group whose Lie algebra $\mathfrak{d}$ can be decomposed into double cross sum [14] of Lie subalgebras $\mathfrak{g}$ and $\tilde{\mathfrak{g}}$ that are maximally isotropic with respect to non-degenerate symmetric bilinear ad-invariant form $\langle.$, . $\rangle$. Drinfel'd double with so-called Manin triple $(\mathfrak{d}, \mathfrak{g}, \tilde{\mathfrak{g}})$ and Lie groups $\mathscr{G}, \widetilde{\mathscr{G}}$ corresponding to $\mathfrak{g}$, $\tilde{\mathfrak{g}}$ is denoted by $\mathscr{D}=(\mathscr{G} \mid \widetilde{\mathscr{G}})$. Poisson-Lie dualizable sigma models on $\mathscr{N} \times \mathscr{G}$ are given by tensor field $\mathcal{F}$ of the form ${ }^{1}$

$$
\mathcal{F}(s, x)=\mathcal{E}(x) \cdot\left(\mathbf{1}_{n+d}+E(s) \cdot \Pi(x)\right)^{-1} \cdot E(s) \cdot \mathcal{E}^{T}(x)
$$

where $E(s)$ is spectator-dependent $(n+d) \times(n+d)$ matrix. Denoting generators of $\mathfrak{g}$ and $\tilde{\mathfrak{g}}$ as $T$ and $\widetilde{T}$, matrix $\Pi(x)$ is given by submatrices $a(x)$ and $b(x)$ of the adjoint representation

$\operatorname{ad}_{g^{-1}}(\widetilde{T})=b(x) \cdot T+a^{-1}(x) \cdot \widetilde{T}$

as

$\Pi(x)=\left(\begin{array}{cc}\mathbf{0}_{n} & 0 \\ 0 & b(x) \cdot a^{-1}(x)\end{array}\right)$.

Matrix $\mathcal{E}(x)$ reads

$\mathcal{E}(x)=\left(\begin{array}{cc}\mathbf{1}_{n} & 0 \\ 0 & e(x)\end{array}\right)$

where $e(x)$ is $d \times d$ matrix of components of right-invariant Maurer-Cartan form $(d g) g^{-1}$ on $\mathscr{G}$.

For many Drinfel'd doubles several decompositions may exist. Suppose that we have sigma model on $\mathscr{N} \times \mathscr{G}$ and the Drinfel'd double splits into another pair of subgroups $\widehat{\mathscr{G}}$

${ }^{1}$ Instead of $\mathcal{F}$ other authors may use its transposition. and $\overline{\mathscr{G}}$. Then we can apply the full framework of PoissonLie T-plurality [1,2] and find background for sigma model on $\mathscr{N} \times \widehat{\mathscr{G}}$.

Let Manin triples $(\mathfrak{d}, \mathfrak{g}, \tilde{\mathfrak{g}})$ and $(\mathfrak{d}, \hat{\mathfrak{g}}, \overline{\mathfrak{g}})$ be two decompositions of $\mathfrak{d}$ into double cross sum of subalgebras that are maximally isotropic with respect to $\langle.,$.$\rangle . Pairs of mutually dual$ bases $T_{a} \in \mathfrak{g}, \widetilde{T}^{a} \in \tilde{\mathfrak{g}}$ and $\widehat{T}_{a} \in \hat{\mathfrak{g}}, \bar{T}^{a} \in \overline{\mathfrak{g}}, a=1, \ldots, d$, then must be related by transformation

$$
\left(\begin{array}{l}
\widehat{T} \\
\bar{T}
\end{array}\right)=C \cdot\left(\begin{array}{l}
T \\
\widetilde{T}
\end{array}\right)
$$

where $C$ is an invertible $2 d \times 2 d$ matrix. For the following formulas it will be convenient to introduce $d \times d$ matrices $P, Q, R, S$ as

$$
\left(\begin{array}{l}
T \\
\widetilde{T}
\end{array}\right)=C^{-1} \cdot\left(\begin{array}{l}
\widehat{T} \\
\bar{T}
\end{array}\right)=\left(\begin{array}{ll}
P & Q \\
R & S
\end{array}\right) \cdot\left(\begin{array}{l}
\widehat{T} \\
\bar{T}
\end{array}\right)
$$

and extend these to $(n+d) \times(n+d)$ matrices

$$
\begin{aligned}
\mathcal{P} & =\left(\begin{array}{cc}
\mathbf{1}_{n} & 0 \\
0 & P
\end{array}\right), \quad \mathcal{Q}=\left(\begin{array}{cc}
\mathbf{0}_{n} & 0 \\
0 & Q
\end{array}\right), \quad \mathcal{R}=\left(\begin{array}{cc}
\mathbf{0}_{n} & 0 \\
0 & R
\end{array}\right), \\
\mathcal{S} & =\left(\begin{array}{cc}
\mathbf{1}_{n} & 0 \\
0 & S
\end{array}\right)
\end{aligned}
$$

to accommodate the spectator fields.

The sigma model on $\mathscr{N} \times \widehat{\mathscr{G}}$ obtained from (3) via Poisson-Lie T-plurality is given by tensor field

$\widehat{\mathcal{F}}(s, \hat{x})=\widehat{\mathcal{E}}(\hat{x}) \cdot \widehat{E}(s, \hat{x}) \cdot \widehat{\mathcal{E}}^{T}(\hat{x}), \quad \widehat{\mathcal{E}}(\hat{x})=\left(\begin{array}{cc}\mathbf{1}_{n} & 0 \\ 0 & \widehat{e}(\hat{x})\end{array}\right)$,

where $\widehat{e}(\hat{x})$ is $d \times d$ matrix of components of right-invariant Maurer-Cartan form $(d \hat{g}) \hat{g}^{-1}$ on $\widehat{\mathscr{G}}$ and

$\widehat{E}(s, \hat{x})=\left(\mathbf{1}_{n+d}+\widehat{E}(s) \cdot \widehat{\Pi}(\hat{x})\right)^{-1} \cdot \widehat{E}(s)=\left(\widehat{E}^{-1}(s)+\widehat{\Pi}(\hat{x})\right)^{-1}$.

The matrix $\widehat{E}(s)$ is obtained from $E(s)$ in (3) by formula

$\widehat{E}(s)=(\mathcal{P}+E(s) \cdot \mathcal{R})^{-1} \cdot(\mathcal{Q}+E(s) \cdot \mathcal{S})$,

and

$$
\begin{aligned}
\widehat{\Pi}(\hat{x}) & =\left(\begin{array}{cc}
\mathbf{0}_{n} & 0 \\
0 & \widehat{b}(\hat{x}) \cdot \widehat{a}^{-1}(\hat{x})
\end{array}\right), \\
\operatorname{ad}_{\hat{g}^{-1}}(\bar{T}) & =\widehat{b}(\hat{x}) \cdot \widehat{T}+\widehat{a}^{-1}(\hat{x}) \cdot \bar{T} .
\end{aligned}
$$

Conformal invariance up to the first loop requires introduction of dilaton field $\Phi$ satisfying beta function equations

$$
\begin{aligned}
& 0=R_{\mu \nu}-\frac{1}{4} H_{\mu \rho \sigma} H_{\nu}{ }^{\rho \sigma}+2 \nabla_{\mu} \nabla_{\nu} \Phi, \\
& 0=-\frac{1}{2} \nabla^{\rho} H_{\rho \mu \nu}+\nabla^{\rho} \Phi H_{\rho \mu \nu}, \\
& 0=R-\frac{1}{12} H_{\rho \sigma \tau} H^{\rho \sigma \tau}+4 \nabla_{\mu} \nabla^{\mu} \Phi-4 \nabla_{\mu} \Phi \nabla^{\mu} \Phi
\end{aligned}
$$


where

$H_{\rho \mu \nu}=\partial_{\rho} \mathcal{B}_{\mu \nu}+\partial_{\mu} \mathcal{B}_{\nu \rho}+\partial_{\nu} \mathcal{B}_{\rho \mu}$,

and $\nabla_{\mu}$ are covariant derivatives with respect to metric $\mathcal{G}$.

Formula for transformation of dilaton under Poisson-Lie T-plurality was given in [2] as

$\widehat{\Phi}(\hat{x})=\Phi(y)+\frac{1}{2} L(y)-\frac{1}{2} \widehat{L}(\hat{x})$

where $y$ represent coordinates of group $\mathscr{G}, \Phi(y)$ is the dilaton of the initial model, and terms $L(y), \widehat{L}(\hat{x}) \mathrm{read}$

$L(y)=\ln |\operatorname{det}[(\mathbf{1}+\Pi(y) E(s)) a(y)]|$,
$\widehat{L}(\hat{x})=\ln \left|\operatorname{det}\left[(\mathbf{1}+\widehat{\Pi}(\hat{x}) \widehat{E}(s)) N^{-1} \widehat{a}(\hat{x})\right]\right|$

with

$N=\mathcal{P}^{T}-\mathcal{R}^{T} E(s)$.

The relation between original and new dilaton can be equivalently written as in $[7,8,15]$ as

$$
\begin{aligned}
& \exp \left(-2 \Phi^{0}(y)\right):=\exp (-2 \Phi(y)) \frac{(\operatorname{det} \mathcal{G}(y))^{1 / 2}}{\operatorname{det} u(y)} \\
& =\exp \left(-2 \widehat{\Phi}^{0}(\hat{x})\right):=\exp (-2 \widehat{\Phi}(\hat{x})) \frac{(\operatorname{det} \widehat{\mathcal{G}}(\hat{x}))^{1 / 2}}{\operatorname{det} \hat{u}(\hat{x})}
\end{aligned}
$$

where $\mathcal{G}$ and $\widehat{\mathcal{G}}$ are metrics of sigma models on $\mathscr{G}$ resp. $\widehat{\mathscr{G}}$ and $u, \hat{u}$ are corresponding matrices of components of leftinvariant forms. The fact that $\Phi^{0}(y)=\widehat{\Phi}^{0}(\hat{x})$ actually allows one to find relation between original and transformed dilaton. Note that $\Phi^{0}(y)=\Phi(y)+\frac{1}{2} L(y)$.

\section{Poisson-Lie transformations and generalized supergravity equations}

In the case that the initial dilaton $\Phi(y)$ depends on coordinates $y^{k}$, we have to express these in terms of $\hat{x}$ and $\bar{x}$ to get explicit form of transformed dilaton. This can be done using relation between two different decompositions of Drinfel'd double elements

$g(y) \tilde{h}(\tilde{y})=\widehat{g}(\hat{x}) \bar{h}(\bar{x}), \quad g \in \mathscr{G}, \tilde{h} \in \tilde{\mathscr{G}}, \widehat{g} \in \widehat{\mathscr{G}}, \bar{h} \in \overline{\mathscr{G}}$.

The origin of the puzzle discussed in [3] is that if

$\frac{\partial y^{k}}{\partial \bar{x}_{j}} \neq 0$

formulas (13) and (14) give $\widehat{\Phi}$ that may depend not only on coordinates $\hat{x}$ of the group $\widehat{\mathscr{G}}$ but also on coordinates $\bar{x}$ of $\overline{\mathscr{G}}$.
Partial solution of this problem was given in [5] for the case of linear dependence

$y^{k}(\hat{x}, \bar{x})=\hat{d}^{k}{ }_{j} \hat{x}^{j}+\bar{d}^{k j} \bar{x}_{j}$.

It was suggested that in this case we can set $y^{k}=\hat{d}^{k}{ }_{m} \hat{x}^{m}$ in the formula (13) and extend the beta function equations to (NS-NS) generalized supergravity equations $[6,16]$

$0=R_{\mu \nu}-\frac{1}{4} H_{\mu \rho \sigma} H_{\nu}{ }^{\rho \sigma}+\nabla_{\mu} X_{\nu}+\nabla_{\nu} X_{\mu}$,

$0=-\frac{1}{2} \nabla^{\rho} H_{\rho \mu \nu}+X^{\rho} H_{\rho \mu \nu}+\nabla_{\mu} X_{v}-\nabla_{\nu} X_{\mu}$,

$0=R-\frac{1}{12} H_{\rho \sigma \tau} H^{\rho \sigma \tau}+4 \nabla_{\mu} X^{\mu}-4 X_{\mu} X^{\mu}$

where

$X_{\mu}=\partial_{\mu} \Phi+\mathcal{J}^{v} \mathcal{F}_{\nu \mu}$

$\Phi$ is the dilaton and $\mathcal{J}$ is a vector field. ${ }^{2}$ For vanishing $\mathcal{J}$ the usual beta function equations are recovered. On the other hand, for backgrounds with $H_{\rho \mu \nu}=0$ Eq. (18) implies that one can find $\Phi^{\prime}$ such that $X_{\mu}=\partial_{\mu} \Phi^{\prime}$. Even though vector field $\mathcal{J}$ introduced in [6] needs to be Killing field, we do not require this property here as it can be changed by gauge transformations keeping $X$ invariant.

Authors of [5,9] give formula allowing to find components of $\widehat{\mathcal{J}}$ for Poisson-Lie transformed sigma model as

$\widehat{\mathcal{J}}^{\alpha}=0, \quad \alpha=1, \ldots, n=\operatorname{dim} \mathscr{N}$,

$\widehat{\mathcal{J}}^{\operatorname{dim} \mathscr{N}+m}(\hat{x})=\left(\frac{1}{2} \bar{f}^{a b}{ }_{b}-\left.\frac{\partial \Phi^{0}(y)}{\partial y^{k}}\right|_{y=\widehat{D} \cdot \hat{x}} \bar{d}^{k a}\right) \widehat{V}_{a}^{m}(\hat{x})$,

where $a, b, k, m=1, \ldots, \operatorname{dim} \mathscr{G}$,

$\widehat{D}=\left(\begin{array}{cc}\mathbf{1}_{n} & 0 \\ 0 & \hat{d}\end{array}\right)$

$\hat{d}$ is the matrix of coefficients $\hat{d}^{k}{ }_{j}$ in (16), $\widehat{V}_{a}$ are (spectator extended, see (4)) left-invariant fields of the group $\widehat{\mathscr{G}}$, and $\bar{f}^{b a}{ }_{c}$ are structure constants of the Lie algebra of $\overline{\mathscr{G}}$. This formula works well for non-Abelian T-duality, i.e. for Poisson-Lie transformations of sigma models with isotropic backgrounds constructed by (3) and (4) where Manin triple is $(\mathfrak{d}, \mathfrak{g}, \mathfrak{a})$ and $\mathfrak{a}$ is Abelian algebra. We have noticed that it does not work for backgrounds obtained by the Poisson-Lie transformations starting from $(\mathfrak{d}, \mathfrak{g}, \tilde{\mathfrak{g}})$ where $\tilde{\mathfrak{g}}$ is neither Abelian nor unimodular, i.e. when $\tilde{f}^{a b}{ }_{b} \neq 0$. Examples given below are backgrounds obtained from $(\mathfrak{d}, \mathfrak{a}, \mathfrak{b})$ where $\mathfrak{b}$ is Bianchi algebra and $\mathfrak{a}$ represents three dimensional Abelian Lie algebra.

2 The Eqs. (17)-(19) without reference to (20) were derived in [17] using connection with torsion. 
From the form of Eqs. (17)-(19) one can see that only oneform $X$ is important for their satisfaction, not separately dilaton $\Phi$ and vector field $\mathcal{J}$. Therefore, for Poisson-Lie transformation of solutions of generalized supergravity equations it would be sufficient to know only Poisson-Lie transformation of the one-form $X$ and of the tensor $\mathcal{F}$.

Note that $\Phi$ and $\mathcal{J}$ are not defined uniquely as the form $X$ is invariant with respect to gauge transformation

$\Phi(x) \mapsto \Phi(x)+\lambda(x), \quad \mathcal{J}^{\kappa} \mapsto \mathcal{J}^{\kappa}-\partial_{\nu} \lambda \mathcal{F}^{\nu \kappa}$

where $\mathcal{F}^{\nu \kappa}=\left(\mathcal{F}^{-1}\right)^{\nu \kappa}$. This means that we can always choose dilaton vanishing. On the other hand, if $X$ is closed, we can choose $X=d \Phi$ and $\mathcal{J}$ vanishing in which case generalized supergravity equations of motion become usual beta function equations. Moreover, note that even the form $X$ satisfying Eqs. (17)-(19) is not unique. Namely, if $X_{\mu}$ satisfy the generalized supergravity equations, then

$X_{\mu}^{\prime}:=X_{\mu}+\chi_{\mu}$,

where

$\nabla_{\nu} \chi_{\mu}=0, \quad\left(X_{\mu}+2 \chi_{\mu}\right) \chi^{\mu}=0$,

satisfy the equations as well. Simple example that was mentioned in [13] is sigma model given by flat Minkowski metric

$\mathcal{F}\left(t, x_{2}, x_{3}\right)=\left(\begin{array}{cccc}1 & 0 & 0 & 0 \\ 0 & 0 & 0 & \frac{1}{x_{3}+1} \\ 0 & 0 & 1 & \frac{x_{2}}{x_{3}+1} \\ 0 & \frac{1}{1-x_{3}} & \frac{x_{2}}{x_{3}-1} & \frac{\left(x_{2}-2\right) x_{2}}{x_{3}^{2}-1}\end{array}\right)$

and

$X_{\mu}=(0,0,0,0), \quad \chi_{\mu}=\left(0,0,0, \frac{1}{1-x_{3}^{2}}\right)$.

In the following we verify whether backgrounds obtained by Poisson-Lie T-plurality supported by $\widehat{\Phi}$ and $\widehat{\mathcal{J}}$ obtained from (13) and (21) satisfy generalized supergravity equations. We give examples where this is not true so that it turns out that formula (21) works well for isometric initial sigma models, but not always. Our original observation was that (21) fails to give solution of generalized supergravity equations in the case of Poisson-Lie T-duality when initial backgrounds are constructed from Manin triple $(\mathfrak{d}, \mathfrak{a}, \tilde{\mathfrak{g}})$ where $\mathfrak{a}$ is abelian and $\tilde{\mathfrak{g}}$ is Bianchi 3, 4 or 5 algebra. Surprisingly, the formula (21) does not work even for Poisson-Lie identity with these Manin triples, i.e. when the $C$ matrix in (5) is identity matrix and the algebraic structure is unaffected. Therefore, it is desirable to modify the prescription for vector fields $\widehat{\mathcal{J}}$. Since no changes are necessary for unimodular algebras $\tilde{\mathfrak{g}}$ in $(\mathfrak{d}, \mathfrak{g}, \tilde{\mathfrak{g}})$, the modified prescription might include terms proportional to $\widetilde{f}^{a b}{ }_{b}$. Gradually searching for more and more involved examples where $\widehat{\mathcal{J}}$ is not only constant but depends on spectators or group coordinates we have found formula

$$
\begin{aligned}
\widehat{\mathcal{J}}^{\operatorname{dim} \mathscr{N}+m}(\hat{x})= & \frac{1}{2} \tilde{f}^{a b}{ }_{b}\left(\frac{\partial \widetilde{y}_{a}}{\partial \bar{x}_{k}} \widehat{V}_{k}{ }^{m}(\hat{x})-\frac{\partial \widetilde{y}_{k}}{\partial \widehat{x}^{a}} \widehat{\mathcal{F}}^{k m}\right) \\
& +\left(\frac{1}{2} \bar{f}^{a b}{ }_{b}-\left.\frac{\partial \Phi^{0}(y)}{\partial y^{k}}\right|_{y=\widehat{D} \cdot \hat{x}} \frac{\partial y^{k}}{\partial \bar{x}_{a}}\right) \widehat{V}_{a}{ }^{m}(\hat{x})
\end{aligned}
$$

where $\widehat{V}_{a}$ are left-invariant fields of the group $\widehat{\mathscr{G}}, \widetilde{f}^{b a}{ }_{c}$ and $\bar{f}^{b a}{ }_{c}$ are structure constants of Lie algebras of $\widetilde{\mathscr{G}}, \overline{\mathscr{G}}$ and $\widehat{D}$ is given by (22). This modification does not change results of $[5,9,18]$ because those papers deal with groups for which $\widetilde{f}^{a b}{ }_{b}=0$. Finally let us mention that $\widehat{\mathcal{J}}$ obtained from (26) is not always Killing vector field of $\widehat{\mathcal{F}}$ (which is not necessary for satisfaction of the NS-NS generalized supergravity equations) but we can use the gauge transformation (24) in order that $\widehat{\mathcal{J}}$ acquire this property.

Having formulas (13), (20) and (26) it is easy to write down prescription for Poisson-Lie transformation of the form $X$

$$
\begin{aligned}
& \widehat{X}_{\mu}(\hat{x})=\left.\frac{\partial \Phi^{0}(y)}{\partial y^{\nu}}\right|_{y=\widehat{D} \cdot \hat{x}} \frac{\partial y^{\nu}}{\partial \hat{x}^{\mu}}-\frac{1}{2} \frac{\partial \widehat{L}(\hat{x})}{\partial \hat{x}^{\mu}}+\widehat{\mathcal{J}}^{\nu}(\hat{x}) \widehat{\mathcal{F}}_{\nu \mu}(\hat{x}) \\
& =\left.\left[X_{\nu}(y)-\mathcal{J}^{\kappa}(y) \mathcal{F}_{\kappa \nu}(y)\right]\right|_{y=\widehat{D} \cdot \hat{x}} \frac{\partial y^{\nu}}{\partial \hat{x}^{\mu}}+\left.\frac{1}{2} \frac{\partial L(y)}{\partial y^{\nu}}\right|_{y=\widehat{D} \cdot \hat{x}} \frac{\partial y^{\nu}}{\partial \hat{x}^{\mu}} \\
& -\frac{1}{2} \frac{\partial \widehat{L}(\hat{x})}{\partial \hat{x}^{\mu}}+\widehat{\mathcal{J}}^{v}(\hat{x}) \widehat{\mathcal{F}}_{v \mu}(\hat{x})
\end{aligned}
$$

where $\widehat{\mathcal{J}}^{v}(\hat{x})$ are given by (26). Advantage of the formula (27) is that $\widehat{X}$, differently from $\widehat{\Phi}$ and $\widehat{\mathcal{J}}$, is invariant with respect to the gauge transformation (23).

\section{Examples}

The difference between formulas (21) and (26) can be shown in examples of Poisson-Lie transformations in Drinfel'd doubles $(\mathscr{A} \mid \widetilde{\mathscr{G}})$ where groups $\widetilde{\mathscr{G}}$ are non-semisimple Bianchi groups. We present transformations in Drinfel'd doubles denoted $^{3}$ as $D D 11$ and $D D 12$ so the examples are given by Poisson-Lie pluralities that follow from series of decompositions

$D D 11:(1 \mid 5) \cong(5 \mid 1) \cong\left(6_{-1} \mid i i 5\right)$,

$D D 12:(1 \mid 4) \cong(4 \mid 1) \cong\left(6_{-1} \mid i i 4\right) \cong\left(i i 4 \mid 6_{-1}\right)$.

Commutation relations defining particular Manin triples and their isomorphisms $C$ are given below. We have also checked

\footnotetext{
3 To denote Drinfel'd doubles and Manin triples we use notation of [19] where classification of six-dimensional Drinfel'd doubles was given. The slots in (.|.) denote numbers corresponding to three dimensional algebras $\mathfrak{g}, \tilde{\mathfrak{g}}$ in Bianchi classification.
} 
pluralities starting with backgrounds on $(2 \mid 5),\left(1 \mid 6_{-1}\right)$ and $\left(6_{-1} \mid i_{i} 5\right)$ as well as Poisson-Lie pluralities in

$D D 13:(1 \mid 3) \cong(3 \mid 1) \cong(3 \mid i i i 3)$

obtaining the same results. Beside physical relevance of sigma models on Bianchi groups that often appear in the literature (see e.g. [5,18] and references therein) the reason for our choice is the fact that in these cases it is possible to solve equations (15) to get the dependence of $y$ and $\tilde{y}$ on $\hat{x}$ and $\bar{x}$. In fact, we have $\frac{\partial \Phi^{0}(y)}{\partial y^{k}}=0$ in the presented examples so we need not to solve the equations (15) and check the linear dependence of $y$ on $\hat{x}$ and $\bar{x}$. Crucial test of the formula (26) would be Poisson-Lie transformation where all terms participate, i.e.

$$
\begin{aligned}
\frac{\partial \Phi^{0}(y)}{\partial y^{k}} & \neq 0, \quad y^{k}(\hat{x}, \bar{x})=\hat{d}_{j}^{k} \hat{x}^{j}+\bar{d}^{k j} \bar{x}_{j}, \quad \tilde{f}_{b}^{a b} \neq 0, \\
\bar{f}^{a b}{ }_{b} & \neq 0 .
\end{aligned}
$$

Unfortunately, it is rather difficult to find such cases.

Group elements will be parametrized as $g=e^{x^{1}} T_{1} e^{x^{2} T_{2}} e^{x^{3} T_{3}}$ where $e^{x^{2} T_{2}} e^{x^{3} T_{3}}$ and $e^{x^{3} T_{3}}$ parametrize their normal subgroups. We deal with backgrounds on four-dimensional manifolds, hence $\operatorname{dim} \mathscr{N}=1$. Spectator $s_{1}$ is denoted as $t$.

\subsection{Poisson-Lie plurality on Drinfel'd double (1|5)}

We shall start our discussion with tensor field ${ }^{4}$

$$
\mathcal{F}(t, y)=\left(\begin{array}{cccc}
-1 & 0 & 0 & 0 \\
0 & \frac{t^{2}}{t^{4}+y_{2}^{2}+y_{3}^{2}} & \frac{y_{2}}{t^{4}+y_{2}^{2}+y_{3}^{2}} & \frac{y_{3}}{t^{4}+y_{2}^{2}+y_{3}^{2}} \\
0 & -\frac{y_{2}}{t^{4}+y_{2}^{2}+y_{3}^{2}} & \frac{t^{4}+y_{3}^{2}}{t^{2}\left(t^{4}+y_{2}^{2}+y_{3}^{2}\right)} & -\frac{y_{2} y_{3}}{t^{2}\left(t^{4}+y_{2}^{2}+y_{3}^{2}\right)} \\
0 & -\frac{y_{3}}{t^{4}+y_{2}^{2}+y_{3}^{2}} & -\frac{y_{2} y_{3}}{t^{2}\left(t^{4}+y_{2}^{2}+y_{3}^{2}\right)} & \frac{t^{4}+y_{2}^{2}}{t^{2}\left(t^{4}+y_{2}^{2}+y_{3}^{2}\right)}
\end{array}\right)
$$

specifying sigma model on Abelian group $\mathscr{G}$ with corresponding Drinfel'd double $\mathscr{D}=(\mathscr{G} \mid \widetilde{\mathscr{G}})=(1 \mid 5)$ whose nontrivial commutation relations read

$$
\begin{array}{rlrl}
{\left[\widetilde{T}^{1}, \widetilde{T}^{2}\right]} & =\widetilde{T}^{2}, & {\left[\widetilde{T}^{1}, \widetilde{T}^{3}\right]=\widetilde{T}^{3},} & {\left[\widetilde{T}^{1}, T_{2}\right]=-T_{2},} \\
{\left[\widetilde{T}^{1}, T_{3}\right]=-T_{3},} & {\left[\widetilde{T}^{2}, T_{2}\right]=T_{1},} & {\left[\widetilde{T}^{3}, T_{3}\right]=T_{1} .}
\end{array}
$$

Background (28), dilaton

$\Phi(t, y)=-\frac{1}{2} \ln \left(-t^{2}\left(t^{4}+y_{2}^{2}+y_{3}^{2}\right)\right)$

and Killing vector $\mathcal{J}=2 \partial_{y_{1}}$ satisfy generalized supergravity equations. Corresponding one-form $X$ with components

$X_{\mu}(t, y)=\frac{1}{t^{4}+y_{2}^{2}+y_{3}^{2}}\left(-\frac{3 t^{4}+y_{2}^{2}+y_{3}^{2}}{t}, 2 t^{2}, y_{2}, y_{3}\right)$

is not closed and we cannot get rid of the vector $\mathcal{J}$ by gauge transformations, so reduction of generalized supergravity equations to beta function equations is not possible.

Background (28) is actually non-Abelian dual of flat background

$\widehat{\mathcal{F}}(t, \hat{x})=\left(\begin{array}{cccc}-1 & 0 & 0 & 0 \\ 0 & t^{2} & 0 & 0 \\ 0 & 0 & e^{2 \hat{x}_{1}} t^{2} & 0 \\ 0 & 0 & 0 & e^{2 \hat{x}_{1}} t^{2}\end{array}\right)$

studied frequently in the literature [18,20,21]. $\widehat{\mathcal{F}}$ is invariant with respect to action of Bianchi 5 group and dilaton (30) and $\mathcal{J}$ were obtained via (13) and (21).

Let us note that $\Phi^{0}(t, y)=-\frac{1}{2} \ln t^{3}$ and it is not necessary to solve equation (15) for $y$ to get transformed dilatons $\widehat{\Phi}(\hat{x})$. Similar results can be obtained starting from Drinfel'd double (1|3).

\subsubsection{Identity $(1 \mid 5) \rightarrow(1 \mid 5)$ and full duality $(1 \mid 5) \rightarrow(5 \mid 1)$}

Let us check formulas (13) and (21) applying Poisson-Lie transformation with $C$ equal to identity matrix to (28) and (30). We recover the original background and dilaton, but vector field $\widehat{\mathcal{J}}=\partial_{y_{1}}$ obtained from (21) is different from the initial one and generalized supergravity equations are not satisfied even in this simple case. Using (26) instead of (21) we get back Killing vector $\widehat{\mathcal{J}}=2 \partial_{y_{1}}$ and generalized supergravity equations are satisfied.

By full duality given by

$C=D_{0}:=\left(\begin{array}{cc}0 & \mathbf{1}_{d} \\ \mathbf{1}_{d} & 0\end{array}\right)$

we get flat background (32), but dilaton

$\widehat{\Phi}(t, \hat{x})=\frac{1}{2} \ln \left(e^{2 \hat{x}_{1}}\right)$

and vanishing vector $\widehat{\mathcal{J}}$ obtained from formulas (13) and (21) do not satisfy generalized supergravity equations.

On the other hand, Eqs. (17)-(19) are satisfied for (32), (34) and

$\widehat{\mathcal{J}}(t, \hat{x})=-\frac{1}{t^{2}} \partial_{\hat{x}_{1}}$

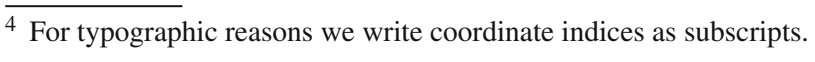


that follows from (26). Corresponding one-form $\widehat{X}$ vanishes, and by gauge transformation it is possible to eliminate $\widehat{\mathcal{J}}$ while changing dilaton to

$$
\widehat{\Phi}^{\prime}(t, \hat{x})=-c_{1} t e^{-\hat{x}_{1}}+c_{2}
$$

with $c_{1}, c_{2}$ arbitrary constants. Dilaton (36) and flat background (32) satisfy beta function equations.

\subsubsection{Plurality $(1 \mid 5) \rightarrow\left(6_{-1} \mid i i 5\right)$}

By Poisson-Lie plurality given by

$C_{(1 \mid 5) \rightarrow\left(6_{-1} \mid i i 5\right)}=\left(\begin{array}{cccccc}0 & 0 & 0 & -1 & 0 & 0 \\ 0 & 0 & 0 & 0 & 1 & 0 \\ 0 & 0 & 1 & 0 & 0 & 0 \\ -1 & 0 & 0 & 0 & 1 & 0 \\ 0 & 1 & 0 & 1 & 0 & 0 \\ 0 & 0 & 0 & 0 & 0 & 1\end{array}\right)$

we get background tensor

$\widehat{\mathcal{F}}(t, \hat{x})=\left(\begin{array}{cccc}-1 & 0 & 0 & 0 \\ 0 & \frac{t^{2} e^{2 \hat{x}_{1}}\left(\hat{x}_{3}^{2}+1\right)}{t^{4}+e^{2 \hat{x}_{1}}\left(\hat{x}_{3}^{2}+1\right)} & \frac{t^{4}}{t^{4}+e^{2 \hat{x}_{1}}\left(\hat{x}_{3}^{2}+1\right)} & \frac{t^{2} e^{2 \hat{x}_{1}} \hat{x}_{3}}{t^{4}+e^{2 \hat{x}_{1}}\left(\hat{x}_{3}^{2}+1\right)} \\ 0 & -\frac{t^{4}}{t^{4}+e^{2 \hat{x}_{1}}\left(\hat{x}_{3}^{2}+1\right)} & \frac{t^{4}}{t^{4}+e^{2 \hat{x}_{1}}\left(\hat{x}_{3}^{2}+1\right)} & \frac{e^{2 \hat{x}_{1}} \hat{x}_{3}}{t^{4}+e^{2 \hat{x}_{1}}\left(\hat{x}_{3}^{2}+1\right)} \\ 0 & \frac{t^{2} e^{2 \hat{x}_{1}} \hat{x}_{3}}{t^{4}+e^{2 \hat{x}_{1}}\left(\hat{x}_{3}^{2}+1\right)} & -\frac{e^{2 \hat{x}_{1}} \hat{x}_{3}}{t^{4}+e^{2 \hat{x}_{1}}\left(\hat{x}_{3}^{2}+1\right)} & \frac{e^{2 \hat{x}_{1}}\left(t^{4}+e^{2 \hat{x}_{1}}\right)}{t^{2} e^{2 \hat{x}_{1}}\left(\hat{x}_{3}^{2}+1\right)+t^{6}}\end{array}\right)$

and dilaton

$\widehat{\Phi}(t, \hat{x})=-\frac{1}{2} \ln \left(t^{2} e^{-2 \hat{x}_{1}}\left(t^{4}+e^{2 \hat{x}_{1}}\left(\hat{x}_{3}^{2}+1\right)\right)\right)$.

Generalized supergravity equations are satisfied for

$\widehat{\mathcal{J}}(t, \hat{x})=\frac{1}{t^{2}} \partial_{\hat{x}_{1}}$

calculated via (26). Vector field $\widehat{\mathcal{J}}=\partial_{\hat{x}_{2}}$ obtained from formula (21) does not satisfy generalized supergravity equations.

Correct one-form $\widehat{X}$ with components

$$
\begin{aligned}
\widehat{X}_{\mu}(t, \hat{x})= & \frac{1}{\Delta}\left(-\frac{3 t^{4}+e^{2 \hat{x}_{1}}\left(\hat{x}_{3}^{2}+1\right)}{t}, e^{2 \hat{x}_{1}}\left(\hat{x}_{3}^{2}+1\right), 2 t^{2}, e^{2 \hat{x}_{1}} \hat{x}_{3}\right), \\
\Delta & =t^{4}+e^{2 \hat{x}_{1}}\left(\hat{x}_{3}^{2}+1\right),
\end{aligned}
$$

is not closed and generalized supergravity equations cannot be reduced to beta function equations. Beside that, vector field (40) is not Killing of (38). However, using the gauge transformation (24) with $\lambda=\hat{x}_{1}$ we get $\widehat{\Phi}^{\prime}(t, \hat{x})=\widehat{\Phi}(t, \hat{x})+$ $\hat{x}_{1}$ and

$\widehat{\mathcal{J}}^{\prime}(t, \hat{x})=2 \partial_{\hat{x}_{2}}$

that is Killing vector field of (38). One-form $\widehat{X}$ remains unchanged, of course.
4.2 Poisson-Lie plurality on Drinfel'd double $(1 \mid 4)$

Next we shall investigate plural sigma models on Drinfel'd double (1|4) with commutation relations

$$
\begin{aligned}
& {\left[\widetilde{T}^{1}, \widetilde{T}^{2}\right]=-\widetilde{T}^{2}+\widetilde{T}^{3}, \quad\left[\widetilde{T}^{1}, \widetilde{T}^{3}\right]=-\widetilde{T}^{3}, \quad\left[\widetilde{T}^{1}, T_{2}\right]=T_{2},} \\
& {\left[\widetilde{T}^{1}, T_{3}\right]=-T_{2}+T_{3}, \quad\left[\widetilde{T}^{2}, T_{2}\right]=-T_{1},} \\
& {\left[\widetilde{T}^{2}, T_{3}\right]=T_{1}, \quad\left[\widetilde{T}^{3}, T_{3}\right]=-T_{1} .}
\end{aligned}
$$

Background

$\mathcal{F}(t, y)=\left(\begin{array}{cccc}1 & 0 & 0 & 0 \\ 0 & 0 & 0 & \frac{1}{1-y_{3}} \\ 0 & 0 & 1 & \frac{y_{3}-y_{2}}{y_{3}-1} \\ 0 & \frac{1}{y_{3}+1} & \frac{y_{3}-y_{2}}{y_{3}+1} & \frac{\left(y_{2}-y_{3}\right)^{2}}{y_{3}^{2}-1}\end{array}\right)$

on Abelian group $\mathscr{G}$ was obtained as non-abelian T-dual of flat background

$\widehat{\mathcal{F}}(t, \hat{x})=\left(\begin{array}{cccc}1 & 0 & 0 & 0 \\ 0 & 0 & e^{-\hat{x}_{1}} \hat{x}_{1} & e^{-\hat{x}_{1}} \\ 0 & e^{-\hat{x}_{1}} \hat{x}_{1} & e^{-2 \hat{x}_{1}} & 0 \\ 0 & e^{-\hat{x}_{1}} & 0 & 0\end{array}\right)$

that is invariant with respect to the action of Bianchi 4 group. Background (43), dilaton

$\Phi(t, y)=-\frac{1}{2} \ln \left(1-y_{3}^{2}\right)$

and Killing vector $\mathcal{J}=-2 \partial_{y_{1}}$ satisfy generalized supergravity equations. Since $\Phi^{0}(y)=0$, we once again do not need to solve (15) to get the transformed dilaton.

\subsubsection{Identity $(1 \mid 4) \rightarrow(1 \mid 4)$}

To check formulas (21) and (26) we apply Poisson-Lie transformation with $C$ equal to identity matrix to (43) and (45). We get the original background and dilaton. Formula (21) gives vector $-\partial_{\hat{x}_{1}}$, while from (26) we obtain Killing vector $\widehat{\mathcal{J}}=-2 \partial_{\hat{x}_{1}}$. For the former generalized supergravity equations are not satisfied, for the latter they hold.

Corresponding one-form

$\widehat{X}(t, \hat{x})=\frac{\hat{x}_{3}+2}{\hat{x}_{3}^{2}-1} d \hat{x}_{3}$

is closed and we can pass to dilaton

$\widehat{\Phi}^{\prime}(t, \hat{x})=\frac{3}{2} \ln \left(1-\hat{x}_{3}\right)-\frac{1}{2} \ln \left(\hat{x}_{3}+1\right)$

that together with (43) satisfies beta function equations. 


\subsubsection{Full duality $(1 \mid 4) \rightarrow(4 \mid 1)$}

By full duality (1|4) $\rightarrow$ (4|1) we get flat background (44), but non-trivial dilaton

$$
\widehat{\Phi}(t, \hat{x})=\frac{1}{2} \ln \left(e^{-2 \hat{x}_{1}}\right)
$$

obtained from (13) and vanishing vector field $\widehat{\mathcal{J}}$ obtained from (21) do not satisfy generalized supergravity equations. Correct vector field for which these equations are satisfied is

$\widehat{\mathcal{J}}(t, \hat{x})=e^{\hat{x}_{1}} \partial_{\hat{x}_{3}}$

and follows from (26). Corresponding one-form $\widehat{X}$ vanishes and using gauge transformation (23) we can get $\widehat{\mathcal{J}}^{\prime}=0$ and dilaton $\widehat{\Phi}^{\prime}=0$ satisfying beta function equations.

\subsubsection{Plurality $(1 \mid 4) \rightarrow\left(6_{-1} \mid i i 4\right)$}

Changing the decomposition of Drinfel'd double to (6-1 $\mid i i 4)$ using matrix

$C_{(1 \mid 4) \rightarrow\left(6_{-1} \mid i i 4\right)}=\left(\begin{array}{cccccc}0 & 0 & 0 & 1 & 0 & 0 \\ 0 & 0 & 0 & 0 & 0 & -2 \\ 0 & 1 & 0 & 0 & 0 & 0 \\ 1 & 0 & 0 & 0 & 0 & -2 \\ 0 & 0 & -\frac{1}{2} & -1 & 0 & 0 \\ 0 & 0 & 0 & 0 & 1 & 0\end{array}\right)$

we get background

$\widehat{\mathcal{F}}(t, \hat{x})=\left(\begin{array}{cccc}1 & 0 & 0 & 0 \\ 0 & -\frac{e^{2 \hat{x}_{1}}\left(\hat{x}_{1}+2 \hat{x}_{3}\right)^{2}}{e^{2 \hat{x}_{1}}-4} & -\frac{2}{e^{\hat{x}_{1}}-2} & -\frac{e^{2 \hat{x}_{1}}\left(\hat{x}_{1}+2 \hat{x}_{3}\right)}{e^{\hat{x}_{1}}-2} \\ 0 & -\frac{2}{e^{\hat{x}_{1}}+2} & 0 & 0 \\ 0 & \frac{e^{2 \hat{x}_{1}}\left(\hat{x}_{1}+2 \hat{x}_{3}\right)}{e^{\hat{x}_{1}}+2} & 0 & e^{2 \hat{x}_{1}}\end{array}\right)$

and dilaton

$\widehat{\Phi}(t, \hat{x})=\frac{1}{2} \ln \left(-\frac{2 e^{2 \hat{x}_{1}}}{e^{2 \hat{x}_{1}}-4}\right)$.

Generalized supergravity equations are satisfied for background (51), dilaton (52) and vector field

$\widehat{\mathcal{J}}(t, \hat{x})=\left(1-\frac{e^{\hat{x}_{1}}}{2}\right) \partial_{\hat{x}_{2}}$

obtained from (26). One-form $\widehat{X}$ corresponding to (52) and (53)

$\widehat{X}(t, \hat{x})=\left(\frac{e^{\hat{x}_{1}}\left(e^{\hat{x}_{1}}-4\right)}{e^{2 \hat{x}_{1}}-4}\right) d \hat{x}_{1}$ is closed so we can eliminate $\widehat{\mathcal{J}}$ by gauge transformation. Dilaton then reads

$\widehat{\Phi}^{\prime}(t, \hat{x})=\frac{1}{2} \ln \left(\frac{\left(2+e^{\hat{x}_{1}}\right)^{3}}{2-e^{\hat{x}_{1}}}\right)$

and together with (51) satisfies beta function equations.

\subsubsection{Plurality $(1 \mid 4) \rightarrow\left(\right.$ ii $\left.4 \mid 6_{-1}\right)$}

Plurality given by matrix $C_{(1 \mid 4) \rightarrow\left(i i 4 \mid 6_{-1}\right)}=D_{0} \cdot C_{(1 \mid 4) \rightarrow\left(6_{-1} \mid i i 4\right)}$ gives flat and torsionless background

$\widehat{\mathcal{F}}(t, \hat{x})=\left(\begin{array}{cccc}1 & 0 & 0 & 0 \\ 0 & 0 & \frac{3}{1-3 e^{\hat{x}_{2}}} & 0 \\ 0 \frac{1}{e^{\hat{x}_{2}+1}} & \frac{3 e^{4 \hat{x}_{2}} \hat{x}_{3}^{2}+4 \hat{x}_{1}}{2 e^{\hat{x}_{2}}+3 e^{2 \hat{x}_{2}}-1} & \frac{e^{\hat{x}_{2}} \hat{x}_{2}+2 e^{3 \hat{x}_{2}} \hat{x}_{3}}{2 e^{\hat{x}_{2}+2}} \\ 0 & 0 & \frac{3 e^{\hat{x}_{2}} \hat{x}_{2}-6 e^{3 \hat{x}_{2}} \hat{x}_{3}}{2-6 e^{\hat{x}_{2}}} & e^{2 \hat{x}_{2}}\end{array}\right)$

and dilaton

$\widehat{\Phi}(t, \hat{x})=\frac{1}{2} \ln \left(\frac{2 e^{2 \hat{x}_{2}}}{-2 e^{\hat{x}_{2}}-3 e^{2 \hat{x}_{2}}+1}\right)$.

Generalized supergravity equations are satisfied for (56), (57) and Killing vector field

$\widehat{\mathcal{J}}(t, \hat{x})=-\frac{1}{3} \partial_{\hat{x}_{1}}$.

One-form $\widehat{X}$ corresponding to (57) and (58)

$\widehat{X}(t, \hat{x})=\frac{2 e^{\hat{x}_{2}}}{\left(e^{\hat{x}_{2}}+1\right)\left(3 e^{\hat{x}_{2}}-1\right)} d \hat{x}_{2}$

is closed and by gauge transformation to dilaton

$\widehat{\Phi}^{\prime}(t, \hat{x})=\frac{1}{2} \ln \left(\frac{1-3 e^{\hat{x}_{2}}}{1+e^{\hat{x}_{2}}}\right)$.

we get solution of beta function equations.

\section{Conclusions}

It follows from the examples in Sects. 4.1 and 4.2 and many others that formulas (13) and (21) for Poisson-Lie transformations of dilatons and Killing vectors [5,7-9] are not universal in the sense that $\widehat{\Phi}$ and $\widehat{\mathcal{J}}$ together with transformed backgrounds in general satisfy neither beta function equations nor generalized supergravity equations. They work properly for transformations of isometric sigma models based on semiabelian Manin triples $(\mathfrak{d}, \mathfrak{g}, \mathfrak{a})$ but not in other cases. We propose modification (26) of formula (21) giving vector fields $\mathcal{J}$ which together with dilatons given by formula (13) (when (16) holds) satisfy generalized supergravity equations for all presented examples (and many others). 
From the form of NS-NS sector of generalized supergravity equations of motion it is clear that knowledge of one-form $X$ is important for their satisfaction, not separately dilaton $\Phi$ and vector field $\mathcal{J}$ that can be changed by gauge transformation. Therefore, beside the Poisson-Lie transformation of tensor $\mathcal{F}$ it is sufficient to know only the transformation of the form $X$ to keep the generalized supergravity equations satisfied. The corresponding formula (27) was checked as well.

In many examples the form $X$ is closed so we can choose $\mathcal{J}$ vanishing by gauge transformation (23), and generalized supergravity equations of motion become usual beta function equations. Beside that, the vector fields $\mathcal{J}$ obtained by the modified formula (26) need not be Killing fields of the corresponding background, but the same transformation can be used to restore this property. Resulting dilatons then differ from those obtained from (13).

In the language of (modified) Double Field Theory used in $[5,22]$, where initial backgrounds are assumed to be isometric, transformation rule (21) can be understood as a field redefinition that is necessary for the covariance of DFT equations of motion and the possibility to restore generalized supergravity equations. In the future we would like to find whether formula (26) can be given in terms of DFT as well.

Data Availability Statement This manuscript has no associated data or the data will not be deposited. [Authors' comment: Article is selfcontained and no additional data is necessary.]

Open Access This article is licensed under a Creative Commons Attribution 4.0 International License, which permits use, sharing, adaptation, distribution and reproduction in any medium or format, as long as you give appropriate credit to the original author(s) and the source, provide a link to the Creative Commons licence, and indicate if changes were made. The images or other third party material in this article are included in the article's Creative Commons licence, unless indicated otherwise in a credit line to the material. If material is not included in the article's Creative Commons licence and your intended use is not permitted by statutory regulation or exceeds the permitted use, you will need to obtain permission directly from the copyright holder. To view a copy of this licence, visit http://creativecomm ons.org/licenses/by/4.0/.

Funded by SCOAP ${ }^{3}$.

\section{References}

1. C. Klimčík, P. Ševera, Dual non-Abelian duality and the Drinfeld double. Phys. Lett. B 351, 455 (1995). arXiv:hep-th/9502122

2. R. von Unge, Poisson-Lie T-plurality. JHEP 07, 014 (2002). arXiv:hep-th/0205245

3. L. Hlavatý, L. Šnobl, Poisson-Lie T-plurality of three-dimensional conformally invariant sigma models II: nondiagonal metrics and dilaton puzzle. JHEP 10, 045 (2004). arXiv:hep-th/0408126

4. S. Demulder, F. Hassler, D.C. Thompson, Doubled aspects of generalised dualities and integrable deformations. JHEP 02, 189 (2019). arXiv:1810.11446
5. Y. Sakatani, Type II DFT solutions from Poisson-Lie Tduality/plurality. Prog. Theor. Exp. Phys. 073B04 (2019). arXiv: 1903.12175

6. L. Wulff, A.A. Tseytlin, Kappa-symmetry of superstring sigma model and generalized 10d supergravity equations. JHEP 06, 174 (2016). arXiv: 1605.04884

7. S. Demulder, F. Hassler, D.C. Thompson, An invitation to Poisson-Lie T-duality in double field theory and its applications. arXiv: 1904.09992

8. R. Borsato, L. Wulff, Quantum correction to generalized Tdualities. Phys. Rev. Lett. 125, 201603 (2020). arXiv:2007.07902

9. L. Hlavatý, I. Petr, Poisson-Lie plurals of Bianchi cosmologies and generalized supergravity equations. JHEP 04, 068 (2020). arXiv: 1910.08436

10. L. Hlavatý, I. Petr, V. Štěpán, Poisson-Lie T-plurality with spectators. J. Math. Phys. 50, 043504 (2009)

11. E. Alvarez, L. Alvarez-Gaume, J.L.F. Barbon, Y. Lozano, Some global aspects of duality in string theory. Nucl. Phys. B 415, 71 (1994). arXiv:hep-th/9309039

12. J.J. Fernández-Melgarejo, J. Sakamoto, Y. Sakatani, K. Yoshida, T-folds from Yang-Baxter deformations. JHEP 12, 108 (2017). arXiv: 1710.06849

13. L. Hlavatý, I. Petr, T-folds as Poisson-Lie plurals. Eur. Phys. J. C 80, 892 (2020). arXiv:2004.08387

14. S. Majid, Foundations of Quantum Group Theory (Cambridge University Press, Cambridge, 1995)

15. T. Codina, D. Marques, Generalized dualities and higher derivatives. JHEP 10, 002 (2020). arXiv:2007.09494

16. G. Arutyunov, S. Frolov, B. Hoare, R. Roiban, A.A. Tseytlin, Scale invariance of the $\eta$-deformed $A d S_{5} \times S^{5}$ superstring, T-duality and modified type II equations. Nucl. Phys. B 903, 262 (2016). arXiv: 1511.05795

17. W. Mück, Generalized supergravity equations and generalized Fradkin-Tseytlin counterterm. JHEP 05, 063 (2019). arXiv:1904.06126

18. M. Honga, Y. Kima, E.Ó. Colgáin, On non-Abelian T-duality for non-semisimple groups. Eur. Phys. J. C 78, 1025 (2018). arXiv: 1801.09567

19. L. Šnobl, L. Hlavatý, Classification of 6-dimensional real Drinfel'd doubles. Int. J. Mod. Phys. A 17, 4043 (2002). arXiv:math.QA/0202209

20. M. Gasperini, R. Ricci, G. Veneziano, A problem with non-Abelian duality? Phys. Lett. B 319, 438 (1993). arXiv:hep-th/9308112

21. E. Álvarez, L. Álvarez-Gaumé, Y. Lozano, On non-abelian duality. Nucl. Phys. B 424, 155 (1994). arXiv:hep-th/9403155v4

22. Y. Sakatani, S. Uehara, K. Yoshida, Generalized gravity from modified DFT. JHEP 1704, 123 (2017). arXiv:1611.05856 